\title{
ROLE OF BEE POLLEN AND SILYMARIN IN AMELIORATION OF PROXIMAL RENAL TUBULAR ACIDOSIS CAUSED BY CISPLATIN
}

\author{
AZIZA A.M. EL-SHAFEY; MOSHIRA M.E. SELIEM; EMAN M.S. SHAHEN; \\ DOAA S. IBRAHIM and DOAA I.M. MAHMOUD \\ Department of Zoology, Faculty of Science, Benha University, Egypt. fax: +2 0133222578.
}

Received: 7 March 2019; Accepted: 31 March 2019

\begin{abstract}
Proximal renal tubular acidosis (RTA), a disorder in acid-base balance in the kidney is characterized by loss of bicarbonate ions in urine. One of its causes is the production of free radicals by cisplatin (CP). Therefore, the protective effects of silymarin and bee pollen as antioxidant natural product treatments were evaluated against proximal RTA induced by CP in male albino rats. Rats were divided into 8 groups ( 7 rats each); control group, cisplatin group, bee pollen group, silymarin group, cisplatin then bee pollen group, cisplatin then silymarin group, bee pollen then cisplatin group and silymarin then cisplatin group. After 12 days (the experiment period), sera were used to determine kidney function parameters (urea, uric acid, and creatinine), protein parameters (total protein, albumin, and globulin), and blood glucose level. While anticoagulant was added to the blood to determine acid-base balance and hematological parameters. Silymarin and bee pollen improved the kidney dysfunction in the CP rats group through decreasing levels of kidney function parameters and increasing levels of serum proteins. They also returned the blood glucose level and the hematological parameters in the CP rats group to the normal values. While the acid-base balance parameters were ameliorated after using silymarin and bee pollen against $\mathrm{CP}$ nephrotoxicity. So, we conclude that silymarin and bee pollen can be used to ameliorate the proximal RTA and anemia induced by CP.
\end{abstract}

Key words: Silymarin, Bee pollen, kidney function, hematological parameters, Cisplatin.

\section{INTRODUCTION}

Renal tubular acidosis (RTA) is a disorder in the acid-base balance in which the kidney loses its ability to excrete enough acid or reabsorb enough bicarbonate $\left(\mathrm{HCO}_{3}{ }^{-}\right)$resulting in metabolic acidosis (Soleimani and Rastegar, 2016). There are four types of RTA; distal RTA (Type 1), proximal RTA (Type 2 ), combined proximal and distal RTA (Type 3) and hyperkalemic RTA (Type 4) (Philip and Jacob, 2018). In type 2 RTA, most bicarbonate ions are lost in urine due to the inability of proximal tubule to reabsorb them from the glomerular filtrate (Yaxley and Pirrone, 2016).

One of the causes of proximal RTA is taking drugs that have a toxic effect on the proximal tubules (Hall et al., 2014). Cisplatin (anticancer drug, CP) is famous for its toxic effect on the proximal tubule (Kitterer et al., 2015). It has been suggested that reactive oxygen species (ROS) induced by cisplatin is one of its toxicity ways (Hosseini et al., 2018). Hayati

Corresponding author: Dr. DOAA S. IBRAHIM E-mail address: Doaa.mohamed@fsc.bu.edu.eg Present address: Department of Zoology, Faculty of Science, Benha University, Egypt. fax: +2 0133222578. et al. (2016) confirmed the necessity of natural antioxidant compounds usage in preventing cisplatin nephrotoxicity. Silymarin and bee pollen are natural products rich in antioxidants and used in the treatment of many diseases (Kocot et al., 2018 and Abouzeinab, 2015). Therefore, we wanted to determine if administration of silymarin and bee pollen have effects on improving proximal RTA.

\section{MATERIALS AND METHODS}

\section{Cisplatin:}

Cisplatin (code number, 171004032) was obtained from Qilu Pharmaceutical Company (Jinan Shandong province, China). It was injected intraperitoneally (single dose, $7 \mathrm{mg} / \mathrm{kg} \mathrm{b.w}$ ) dissolved in $0.5 \mathrm{ml}$ water (Mansour et al., 2006).

\section{Bee Pollen:}

Bee Pollen was obtained from the honeybee project, Faculty of Agriculture, Benha University, Egypt. It was administered orally (daily dose, $300 \mathrm{mg} / \mathrm{kg} \mathrm{b.w)}$ dissolved in $0.5 \mathrm{ml}$ water (Yildiz et al., 2013).

\section{Silymarin:}

Silymarin was obtained as a drug (each capsule contain $140 \mathrm{mg}$ of pure silymarin) from Chemical 
Industries Development (CID) Company (Giza, Egypt). It was administrated orally (daily dose, 100 $\mathrm{mg} / \mathrm{kg} \mathrm{b.w}$ ) dissolved in $0.5 \mathrm{ml}$ water (Mohamed et al., 2015).

\section{Animals:}

Fifty-six rats male albino rats $(155 \pm 20 \mathrm{~g})$ were obtained from the VSVRI (Veterinary Serum and Vaccine Research Institute, Abbasia, Cairo, Egypt). Rats were housed in wired cages and kept under constant environmental conditions $\left(25 \pm 2^{\circ} \mathrm{C}\right.$ and $12 \mathrm{hr}$ dark/light cycle), fed on a standard diet and supplied with water ad libitum throughout the experimental period.

\section{Animal groups:}

Rats were divided into eight groups (7 rats each); control group (normal untreated rats), cisplatin group (rats injected with cisplatin one time), bee pollen group (rats administrated bee pollen for 12 days), silymarin group (rats administrated silymarin for 12 days), cisplatin then bee pollen group (rats injected with cisplatin then after 5 days treated with a daily oral dose of bee pollen for 7 days), cisplatin then silymarin group (rats injected with cisplatin then after 5 days treated with a daily oral dose of silymarin for 7 days), bee pollen then cisplatin group (rats administrated a daily oral dose of bee pollen for 7 days then injected with cisplatin) and silymarin then cisplatin group (rats administrated silymarin for 7 days then injected with cisplatin). Experimental period was extended for 12 days.

\section{Blood Samples collection and preparation:}

At the end of experiment period, all fasted overnight rats were anesthetized. Blood samples were collected from posterior vena cava in two groups of tubes, the first tubes group were containing EDTA as an anticoagulant for determination of hematological parameters while the blood in the second tubes group allowed to clot without using any anticoagulants 1-2 $\mathrm{h}$ at $37^{\circ} \mathrm{C}$ then centrifuged at $3000 \mathrm{rpm}$ for 15 minutes. Sera were separated and stored at $-20{ }^{\circ} \mathrm{C}$ for biochemical determinations. Arterial and venous blood samples of each group were collected into 1.0 $\mathrm{ml}$ syringes containing heparin $(5000 \mathrm{IU} / \mathrm{ml})$ from the dorsal aorta and posterior vena cava for determinations of acid-base balance parameters.

\section{Biochemical, acid-base balance and hematological parameters:}

Serum urea, uric acid, and creatinine levels were determined spectrophotometrically by using Diamond, Spinreact and Diamond kits, respectively.

Serum total protein and albumin levels were determined spectrophotometrically by using Diamond kits. While serum globulin level was calculated as follows:

Globulin $=$ Total protein - Albumin
Glucose was determined spectrophotometrically by using Spinreact kit.

Acid-base balance parameters (potential of hydrogen "pH" value, carbon dioxide partial pressure " $\mathrm{PCO}_{2}$ ", bicarbonate $" \mathrm{HCO}_{3}{ }^{-1}$, total carbon dioxide $" \mathrm{TCO}_{2} "$ and base excess "BE") were determined in arterial and venous blood samples using MEDICA, REF7001, Analyzer, English.

Hematological parameters (hemoglobin "Hb" content, hematocrit "Hct" value, red blood cells "RBCs" count, mean corpuscular volume "MCV", mean corpuscular hemoglobin "MCH", mean corpuscular hemoglobin concentration "MCHC", white blood cells "WBCs" count and blood platelets count) were determined by automated hematology cell counter (MS4e Automatic Hematology Analyzer).

Statistical Analysis: All measured and calculated parameter values were expressed as the mean of seven individual values \pm standard error "SE". The computer program Statistical Package for Social Science (SPSS) (Version 20.00, Chicago, USA) was used for performing one-way analysis of variance (ANOVA) followed by Duncan's multiple range test.

\section{RESULTS}

The kidney function parameters (urea, uric acid, and creatinine) levels showed significant increases $(\mathrm{P}<0.05)$ in the $\mathrm{CP}$ group compared to control, bee pollen and silymarin groups. All treated CP groups (cisplatin then bee pollen, cisplatin then silymarin, bee pollen then cisplatin and silymarin then cisplatin) have low levels of kidney function parameters in comparison with CP group (Table 1).

The protein parameters (total protein, albumin, and globulin) levels in the CP group showed significant decreases $(\mathrm{P}<0.05)$ compared to control, bee pollen and silymarin groups. while protein parameters levels in all treated CP groups increased significantly (Table 2).

Blood glucose in the CP group increased significantly $(\mathrm{P}<0.05)$ compared to other groups. whereas all treated $\mathrm{CP}$ groups showed significant decreases in blood glucose levels (Figure 1).

Both arterial and venous blood acid-base balance parameters $\left(\mathrm{pH}, \mathrm{PCO}_{2}, \mathrm{HCO}_{3}-, \mathrm{TCO}_{2}\right.$ and $\left.\mathrm{BE}\right)$ in the $\mathrm{CP}$ group decreased significantly $(\mathrm{P}<0.05)$ compared to control, bee pollen and silymarin groups, while in all treated CP groups they increased significantly in arteries and veins except the arterial $\mathrm{PCO}_{2}$ levels in cisplatin then bee pollen, bee pollen then cisplatin and silymarin then cisplatin groups which showed non- 
significant increase. Only the cisplatin then silymarin group showed significant increase in $\mathrm{PCO}_{2}$ compared to $\mathrm{CP}$ group in its arterial blood (Table 3 ).

Cisplatin lowered the $\mathrm{Hb}$ content, Hct value, RBCs count, and platelets count, but it increased the WBCs count. While the Hb content, Hct value, RBCs count, and platelets count increased significantly and WBCs count decreased significantly in all treated $\mathrm{CP}$ groups compared to the CP group (Table 4).

Table 1: Effects of bee pollen and silymarin on kidney function parameters in different experimental groups.

\begin{tabular}{|c|c|c|c|c|c|c|c|c|}
\hline \multirow{2}{*}{ Parameters } & \multicolumn{8}{|c|}{ Groups } \\
\hline & Control & Cisplatin & $\begin{array}{c}\text { Bee } \\
\text { Pollen }\end{array}$ & Silymarin & $\begin{array}{c}\text { Cisplatin } \\
\text { then Bee } \\
\text { Pollen }\end{array}$ & $\begin{array}{c}\text { Cisplatin } \\
\text { then } \\
\text { Silymarin }\end{array}$ & $\begin{array}{c}\text { Bee Pollen } \\
\text { then } \\
\text { Cisplatin }\end{array}$ & $\begin{array}{c}\text { Silymarin } \\
\text { then } \\
\text { Cisplatin }\end{array}$ \\
\hline $\begin{array}{c}\text { Urea } \\
(\mathrm{mg} / \mathrm{dl})\end{array}$ & $\begin{array}{c}38.60 \pm \\
1.36^{\mathrm{d}}\end{array}$ & $\begin{array}{c}175.80 \pm \\
4.08^{\mathrm{a}}\end{array}$ & $\begin{array}{c}34.80 \pm \\
2.03^{\mathrm{d}}\end{array}$ & $\begin{array}{c}36.00 \pm \\
0.63^{\mathrm{d}}\end{array}$ & $\begin{array}{c}56.20 \pm \\
1.16^{c}\end{array}$ & $\begin{array}{c}62.40 \pm \\
1.28^{b}\end{array}$ & $\begin{array}{c}52.80 \pm \\
0.86^{c}\end{array}$ & $\begin{array}{l}51.60 \pm \\
1.03^{c}\end{array}$ \\
\hline $\begin{array}{l}\text { Uric acid } \\
(\mathrm{mg} / \mathrm{dl})\end{array}$ & $\begin{array}{l}1.22 \pm \\
0.07^{\mathrm{d}}\end{array}$ & $\begin{array}{l}2.45 \pm \\
0.06^{\mathrm{a}}\end{array}$ & $\begin{array}{l}1.08 \pm \\
0.04^{\mathrm{e}}\end{array}$ & $\begin{array}{l}1.22 \pm \\
0.02^{\mathrm{d}}\end{array}$ & $\begin{array}{l}1.86 \pm \\
0.02^{\mathrm{b}}\end{array}$ & $\begin{array}{l}1.82 \pm \\
0.02^{\mathrm{b}}\end{array}$ & $\begin{array}{l}1.68 \pm \\
0.06^{c}\end{array}$ & $\begin{array}{l}1.56 \pm \\
0.09^{c}\end{array}$ \\
\hline $\begin{array}{c}\text { Creatinine } \\
\text { (mg/dl) }\end{array}$ & $\begin{array}{l}0.37 \pm \\
0.01^{\mathrm{d}}\end{array}$ & $\begin{array}{l}2.82 \pm \\
0.03^{\mathrm{a}}\end{array}$ & $\begin{array}{l}0.38 \pm \\
0.02^{\mathrm{d}}\end{array}$ & $\begin{array}{l}0.42 \pm \\
0.01^{\mathrm{d}}\end{array}$ & $\begin{array}{l}1.35 \pm \\
0.08^{\mathrm{b}}\end{array}$ & $\begin{array}{l}1.22 \pm \\
0.11^{\mathrm{b}}\end{array}$ & $\begin{array}{l}0.77 \pm \\
0.02^{c}\end{array}$ & $\begin{array}{l}0.78 \pm \\
0.04^{\mathrm{c}}\end{array}$ \\
\hline
\end{tabular}

Values are expressed as means \pm standard errors for seven rats.

abcde $=$ values in same raw with different small letters are significantly different at $(\mathrm{P}<0.05)$.

Table 2: Effects of bee pollen and silymarin on serum proteins in different experimental groups.

\begin{tabular}{|c|c|c|c|c|c|c|c|c|}
\hline \multirow[t]{2}{*}{ Parameters } & \multicolumn{8}{|c|}{ Groups } \\
\hline & Control & Cisplatin & $\begin{array}{c}\text { Bee } \\
\text { Pollen }\end{array}$ & Silymarin & $\begin{array}{c}\text { Cisplatin } \\
\text { then Bee } \\
\text { Pollen }\end{array}$ & $\begin{array}{l}\text { Cisplatin } \\
\text { then } \\
\text { Silymarin }\end{array}$ & $\begin{array}{c}\text { Bee } \\
\text { Pollen } \\
\text { then } \\
\text { Cisplatin }\end{array}$ & $\begin{array}{c}\text { Silymarin } \\
\text { then } \\
\text { Cisplatin }\end{array}$ \\
\hline \multirow{2}{*}{$\begin{array}{c}\text { Total } \\
\text { protein } \\
\text { (g/dl) }\end{array}$} & $6.72 \pm$ & $4.29 \pm$ & $6.60 \pm$ & $6.68 \pm$ & $5.68 \pm$ & $5.78 \pm$ & $5.72 \pm$ & $5.73 \pm$ \\
\hline & $0.05^{\mathrm{a}}$ & $0.06^{\mathrm{c}}$ & $0.06^{\mathrm{a}}$ & $0.03^{\mathrm{a}}$ & $0.04^{b}$ & $0.02^{b}$ & $0.05^{b}$ & $0.03^{b}$ \\
\hline \multirow{2}{*}{$\begin{array}{l}\text { Albumin } \\
\text { (g/dl) }\end{array}$} & $4.08 \pm$ & $2.70 \pm$ & $3.86 \pm$ & $3.92 \pm$ & $3.51 \pm$ & $3.60 \pm$ & $3.56 \pm$ & $3.57 \pm$ \\
\hline & $0.17^{\mathrm{a}}$ & $0.12^{\mathrm{c}}$ & $0.06^{\mathrm{a}}$ & $0.04^{\mathrm{a}}$ & $0.03^{b}$ & $0.03^{b}$ & $0.04^{b}$ & $0.03^{b}$ \\
\hline \multirow{2}{*}{$\begin{array}{l}\text { Globulin } \\
\text { (g/dl) }\end{array}$} & $2.64 \pm$ & $1.59 \pm$ & $2.74 \pm$ & $2.76 \pm$ & $2.17 \pm$ & $2.18 \pm$ & $2.16 \pm$ & $2.15 \pm$ \\
\hline & $0.20^{\mathrm{a}}$ & $0.14^{\mathrm{c}}$ & $0.02^{\mathrm{a}}$ & $0.05^{\mathrm{a}}$ & $0.06^{b}$ & $0.03^{b}$ & $0.05^{\mathrm{b}}$ & $0.02^{\mathrm{b}}$ \\
\hline
\end{tabular}

Values are expressed as means \pm standard errors for seven rats.

$\mathrm{abc}=$ values in same raw with different small letters are significantly different at $(\mathrm{P}<0.05)$. 
Table 3: Effects of bee pollen and silymarin on acid-base balance parameters in different experimental groups.

\begin{tabular}{|c|c|c|c|c|c|c|c|c|c|}
\hline \multicolumn{6}{|c|}{ Parameters } & \multicolumn{3}{|c|}{ Groups } & \multirow[b]{2}{*}{$\begin{array}{c}\text { Silymarin } \\
\text { then } \\
\text { Cisplatin }\end{array}$} \\
\hline & & Control & Cisplatin & $\begin{array}{c}\text { Bee } \\
\text { Pollen }\end{array}$ & Silymarin & $\begin{array}{c}\text { Cisplatin } \\
\text { then Bee } \\
\text { Pollen } \\
\end{array}$ & $\begin{array}{c}\text { Cisplatin } \\
\text { then } \\
\text { Silymarin }\end{array}$ & $\begin{array}{c}\text { Bee Pollen } \\
\text { then } \\
\text { Cisplatin } \\
\end{array}$ & \\
\hline \multirow{2}{*}{$\underset{\text { (unit) }}{\text { pH }}$} & $\mathbf{A}$ & $\begin{array}{l}7.41 \pm \\
0.02^{\mathrm{a}} \\
\end{array}$ & $\begin{array}{l}7.16 \pm \\
0.01^{\mathrm{d}} \\
\end{array}$ & $\begin{array}{l}7.39 \pm \\
0.02^{\mathrm{a}} \\
\end{array}$ & $\begin{array}{l}7.41 \pm \\
0.00^{\mathrm{a}} \\
\end{array}$ & $\begin{array}{l}7.22 \pm \\
0.01^{\mathrm{c}} \\
\end{array}$ & $\begin{array}{l}7.32 \pm \\
0.01^{\mathrm{b}} \\
\end{array}$ & $\begin{array}{l}7.33 \pm \\
0.00^{\mathrm{b}} \\
\end{array}$ & $\begin{array}{l}7.25 \pm \\
0.00^{\mathrm{c}} \\
\end{array}$ \\
\hline & $\mathbf{V}$ & $\begin{array}{l}7.40 \pm \\
0.01^{\mathrm{a}} \\
\end{array}$ & $\begin{array}{l}7.15 \pm \\
0.03^{\mathrm{c}}\end{array}$ & $\begin{array}{l}7.39 \pm \\
0.01^{\mathrm{a}} \\
\end{array}$ & $\begin{array}{l}7.40 \pm \\
0.00^{\mathrm{a}} \\
\end{array}$ & $\begin{array}{l}7.24 \pm \\
0.01^{\mathrm{b}} \\
\end{array}$ & $\begin{array}{l}7.25 \pm \\
0.01^{\mathrm{b}} \\
\end{array}$ & $\begin{array}{l}7.17 \pm \\
0.00^{\mathrm{c}} \\
\end{array}$ & $\begin{array}{l}7.26 \pm \\
0.00^{\mathrm{b}}\end{array}$ \\
\hline \multirow{2}{*}{$\begin{array}{c}\mathrm{PCO}_{2} \\
(\mathbf{m m H g})\end{array}$} & $\mathbf{A}$ & $\begin{array}{c}48.33 \pm \\
1.66^{\mathrm{a}}\end{array}$ & $\begin{array}{c}32.77 \pm \\
1.67^{\mathrm{c}, \mathrm{d}}\end{array}$ & $\begin{array}{c}46.56 \pm \\
1.71^{\mathrm{a}}\end{array}$ & $\begin{array}{c}45.90 \pm \\
1.27^{\mathrm{a}}\end{array}$ & $\begin{array}{l}37.90 \pm \\
2.01^{\mathrm{b}, \mathrm{c}}\end{array}$ & $\begin{array}{c}43.00 \pm \\
2.37^{\mathrm{a}, \mathrm{b}}\end{array}$ & $\begin{array}{c}31.37 \pm \\
1.94^{\mathrm{d}}\end{array}$ & $\begin{array}{l}35.88 \pm \\
2.82^{\mathrm{c}, \mathrm{d}}\end{array}$ \\
\hline & $\mathbf{V}$ & $\begin{array}{c}64.93 \pm \\
1.09^{\mathrm{a}} \\
\end{array}$ & $\begin{array}{c}35.13 \pm \\
3.64^{\mathrm{c}} \\
\end{array}$ & $\begin{array}{c}63.33 \pm \\
0.88^{\mathrm{a}} \\
\end{array}$ & $\begin{array}{c}64.00 \pm \\
2.08^{\mathrm{a}} \\
\end{array}$ & $\begin{array}{c}48.18 \pm \\
1.06^{\mathrm{b}} \\
\end{array}$ & $\begin{array}{c}46.60 \pm \\
1.12^{\mathrm{b}} \\
\end{array}$ & $\begin{array}{c}45.36 \pm \\
0.98^{\mathrm{b}} \\
\end{array}$ & $\begin{array}{c}47.36 \pm \\
0.52^{\mathrm{b}} \\
\end{array}$ \\
\hline \multirow{2}{*}{$\begin{array}{c}\mathrm{HCO}_{3}^{-} \\
(\mathrm{mmol} / \mathrm{L})\end{array}$} & $\mathbf{A}$ & $\begin{array}{c}30.36 \pm \\
1.7^{\mathrm{a}} \\
\end{array}$ & $\begin{array}{c}11.40 \pm \\
0.75^{\mathrm{d}}\end{array}$ & $\begin{array}{c}27.63 \pm \\
0.24^{\mathrm{a}}\end{array}$ & $\begin{array}{c}28.13 \pm \\
1.12^{\mathrm{a}} \\
\end{array}$ & $\begin{array}{c}14.73 \pm \\
0.87^{\mathrm{c}} \\
\end{array}$ & $\begin{array}{c}21.40 \pm \\
1.35^{\mathrm{b}}\end{array}$ & $\begin{array}{c}15.93 \pm \\
0.82^{\mathrm{c}}\end{array}$ & $\begin{array}{c}15.16 \pm \\
0.97^{\mathrm{c}}\end{array}$ \\
\hline & $\mathbf{V}$ & $\begin{array}{c}38.90 \pm \\
1.46^{\mathrm{a}} \\
\end{array}$ & $\begin{array}{c}11.80 \pm \\
0.43^{\mathrm{d}}\end{array}$ & $\begin{array}{c}37.33 \pm \\
0.98^{\mathrm{a}} \\
\end{array}$ & $\begin{array}{c}38.30 \pm \\
1.47^{\mathrm{a}} \\
\end{array}$ & $\begin{array}{c}20.13 \pm \\
0.60^{\mathrm{b}}\end{array}$ & $\begin{array}{c}19.76 \pm \\
0.72^{\mathrm{b}} \\
\end{array}$ & $\begin{array}{c}16.00 \pm \\
0.50^{\mathrm{c}}\end{array}$ & $\begin{array}{c}20.53 \pm \\
0.43^{\mathrm{b}}\end{array}$ \\
\hline \multirow{2}{*}{$\begin{array}{c}\mathrm{TCO}_{2} \\
(\mathrm{mmol} / \mathrm{L})\end{array}$} & $\mathbf{A}$ & $\begin{array}{c}28.13 \pm \\
1.09^{\mathrm{a}}\end{array}$ & $\begin{array}{c}13.80 \pm \\
1.28^{\mathrm{d}}\end{array}$ & $\begin{array}{l}25.05 \pm \\
2.22^{\mathrm{a}, \mathrm{b}}\end{array}$ & $\begin{array}{c}26.16 \pm \\
2.68^{\mathrm{a}, \mathrm{b}}\end{array}$ & $\begin{array}{c}21.50 \pm \\
0.86^{\mathrm{b}, \mathrm{c}}\end{array}$ & $\begin{array}{l}17.16 \pm \\
1.01^{\mathrm{c}, \mathrm{d}}\end{array}$ & $\begin{array}{c}20.10 \pm \\
0.66^{c}\end{array}$ & $\begin{array}{c}19.15 \pm \\
0.83^{\mathrm{c}} \\
\end{array}$ \\
\hline & $\mathbf{V}$ & $\begin{array}{c}27.80 \pm \\
1.94^{\mathrm{a}, \mathrm{b}}\end{array}$ & $\begin{array}{c}17.10 \pm \\
1.06^{\mathrm{c}}\end{array}$ & $\begin{array}{c}31.00 \pm \\
1.00^{\mathrm{a}}\end{array}$ & $\begin{array}{c}29.15 \pm \\
0.89^{\mathrm{a}, \mathrm{b}}\end{array}$ & $\begin{array}{l}27.10 \pm \\
3.01^{\mathrm{a}, \mathrm{b}} \\
\end{array}$ & $\begin{array}{c}28.06 \pm \\
0.52^{\mathrm{a}, \mathrm{b}}\end{array}$ & $\begin{array}{c}25.00 \pm \\
2.30^{\mathrm{b}} \\
\end{array}$ & $\begin{array}{c}24.86 \pm \\
0.69^{\mathrm{b}} \\
\end{array}$ \\
\hline \multirow{2}{*}{$\begin{array}{c}\text { BE } \\
(\mathrm{mmol} / \mathrm{L})\end{array}$} & $\mathbf{A}$ & $\begin{array}{c}5.77 \pm \\
1.73^{\mathrm{a}} \\
\end{array}$ & $\begin{array}{c}-15.33 \pm \\
0.88^{\mathrm{d}} \\
\end{array}$ & $\begin{array}{c}2.93 \pm 0 . \\
46^{\mathrm{a}} \\
\end{array}$ & $\begin{array}{c}3.60 \pm \\
1.15^{\mathrm{a}} \\
\end{array}$ & $\begin{array}{c}-11.26 \pm \\
0.66^{\mathrm{c}} \\
\end{array}$ & $\begin{array}{c}-3.86 \pm \\
1.36^{\mathrm{b}} \\
\end{array}$ & $\begin{array}{c}-8.83 \pm \\
0.72^{c} \\
\end{array}$ & $\begin{array}{c}-10.66 \pm \\
0.82^{\mathrm{c}} \\
\end{array}$ \\
\hline & $\mathbf{V}$ & $\begin{array}{c}13.47 \pm \\
1.50^{\mathrm{a}} \\
\end{array}$ & $\begin{array}{c}-15.13 \pm \\
0.50^{\mathrm{d}}\end{array}$ & $\begin{array}{c}11.93 \pm \\
0.99^{\mathrm{a}} \\
\end{array}$ & $\begin{array}{c}12.87 \pm \\
1.43^{\mathrm{a}} \\
\end{array}$ & $\begin{array}{c}-6.16 \pm \\
0.66^{\mathrm{b}} \\
\end{array}$ & $\begin{array}{l}-6.4 \pm \\
0.75^{b} \\
\end{array}$ & $\begin{array}{c}-11.00 \pm \\
0.50^{\mathrm{c}} \\
\end{array}$ & $\begin{array}{c}-5.53 \pm \\
0.47^{\mathrm{b}} \\
\end{array}$ \\
\hline
\end{tabular}

Values are expressed as means \pm standard errors for seven rats.

abcd $=$ values in same raw with different small letters are significantly different at $(\mathrm{P}<0.05)$.

$\mathrm{A}=$ artery.

$\mathrm{V}=$ vein.

Table 4: Effects of bee pollen and silymarin on hematological parameters in different experimental groups.

\begin{tabular}{|c|c|c|c|c|c|c|c|c|}
\hline \multicolumn{4}{|c|}{ Parameters } & \multicolumn{5}{|c|}{ Groups } \\
\hline & Control & Cisplatin & Bee Pollen & Silymarin & $\begin{array}{c}\text { Cisplatin } \\
\text { then Bee } \\
\text { Pollen }\end{array}$ & $\begin{array}{l}\text { Cisplatin } \\
\text { then } \\
\text { Silymarin }\end{array}$ & $\begin{array}{c}\text { Bee Pollen } \\
\text { then } \\
\text { Cisplatin }\end{array}$ & $\begin{array}{c}\text { Silymarin } \\
\text { then } \\
\text { Cisplatin }\end{array}$ \\
\hline $\begin{array}{c}\text { Hb } \\
\text { (g/dl) }\end{array}$ & $\begin{array}{l}9.82 \pm \\
0.21^{\mathrm{b}}\end{array}$ & $\begin{array}{l}7.32 \pm \\
0.17^{\mathrm{c}}\end{array}$ & $\begin{array}{c}11.24 \pm \\
0.08^{\mathrm{a}}\end{array}$ & $\begin{array}{c}10.02 \pm \\
0.36^{\mathrm{b}}\end{array}$ & $\begin{array}{l}9.50 \pm \\
0.18^{\mathrm{b}}\end{array}$ & $\begin{array}{r}9.44 \pm \\
0.22^{\mathrm{b}}\end{array}$ & $\begin{array}{c}10.16 \pm \\
0.29^{\mathrm{b}}\end{array}$ & $\begin{array}{r}9.76 \pm \\
0.17^{b}\end{array}$ \\
\hline Hct $(\%)$ & $\begin{array}{c}29.82 \pm \\
0.86^{\mathrm{b}}\end{array}$ & $\begin{array}{c}21.58 \pm \\
0.68^{c}\end{array}$ & $\begin{array}{c}33.40 \pm \\
0.48^{\mathrm{a}}\end{array}$ & $\begin{array}{c}30.96 \pm \\
1.38^{\mathrm{a}, \mathrm{b}} \\
\end{array}$ & $\begin{array}{c}28.14 \pm \\
0.98^{\mathrm{b}}\end{array}$ & $\begin{array}{c}27.88 \pm \\
1.02^{\mathrm{b}} \\
\end{array}$ & $\begin{array}{c}29.68 \pm \\
1.30^{\mathrm{b}} \\
\end{array}$ & $\begin{array}{c}29.08 \pm \\
0.83^{b} \\
\end{array}$ \\
\hline $\begin{array}{c}\text { RBCs } \\
\left(\mathrm{M} / \mathrm{mm}^{3}\right)\end{array}$ & $\begin{array}{c}5.33 \pm \\
0.10^{\mathrm{b}} \\
\end{array}$ & $\begin{array}{r}3.98 \pm \\
0.15^{\mathrm{c}} \\
\end{array}$ & $\begin{array}{l}6.30 \pm \\
0.10^{\mathrm{a}} \\
\end{array}$ & $\begin{array}{l}5.59 \pm \\
0.09^{\mathrm{b}} \\
\end{array}$ & $\begin{array}{l}5.21 \pm \\
0.12^{\mathrm{b}} \\
\end{array}$ & $\begin{array}{l}5.26 \pm \\
0.07^{\mathrm{b}}\end{array}$ & $\begin{array}{c}5.59 \pm \\
0.18^{\mathrm{b}} \\
\end{array}$ & $\begin{array}{c}5.29 \pm \\
0.09^{\mathrm{b}} \\
\end{array}$ \\
\hline $\begin{array}{c}\text { MCV } \\
\text { (fl) }\end{array}$ & $\begin{array}{c}55.84 \pm \\
1.04^{\mathrm{a}}\end{array}$ & $\begin{array}{c}54.16 \pm \\
0.34^{\mathrm{a}}\end{array}$ & $\begin{array}{c}53.00 \pm \\
0.86^{\mathrm{a}}\end{array}$ & $\begin{array}{c}55.30 \pm \\
1.62^{\mathrm{a}}\end{array}$ & $\begin{array}{c}53.98 \pm \\
1.15^{\mathrm{a}}\end{array}$ & $\begin{array}{c}52.94 \pm \\
1.18^{\mathrm{a}}\end{array}$ & $\begin{array}{c}53.06 \pm \\
1.33^{\mathrm{a}}\end{array}$ & $\begin{array}{c}54.90 \pm \\
1.03^{\mathrm{a}} \\
\end{array}$ \\
\hline $\begin{array}{c}\mathrm{MCH} \\
(\mathrm{pg})\end{array}$ & $\begin{array}{c}18.40 \pm \\
0.34^{\mathrm{a}}\end{array}$ & $\begin{array}{c}18.44 \pm \\
0.26^{\mathrm{a}}\end{array}$ & $\begin{array}{c}17.84 \pm \\
0.24^{\mathrm{a}}\end{array}$ & $\begin{array}{c}17.92 \pm \\
0.36^{\mathrm{a}}\end{array}$ & $\begin{array}{c}18.24 \pm \\
0.30^{\mathrm{a}}\end{array}$ & $\begin{array}{c}17.92 \pm \\
0.23^{\mathrm{a}}\end{array}$ & $\begin{array}{c}18.16 \pm \\
0.08^{\mathrm{a}}\end{array}$ & $\begin{array}{c}18.42 \pm \\
0.15^{\mathrm{a}}\end{array}$ \\
\hline $\begin{array}{c}\mathrm{MCHC} \\
(\mathrm{g} / \mathrm{dl})\end{array}$ & $\begin{array}{r}32.96 \pm \\
0.38^{\mathrm{a}, \mathrm{b}}\end{array}$ & $\begin{array}{l}33.98 \pm \\
0.29^{\mathrm{a}, \mathrm{b}}\end{array}$ & $\begin{array}{c}33.67 \pm \\
0.49^{\mathrm{a}, \mathrm{b}}\end{array}$ & $\begin{array}{c}32.44 \pm \\
0.26^{\mathrm{b}}\end{array}$ & $\begin{array}{r}33.84 \pm \\
0.59^{\mathrm{a}, \mathrm{b}}\end{array}$ & $\begin{array}{r}33.92 \pm \\
0.65^{\mathrm{a}, \mathrm{b}}\end{array}$ & $\begin{array}{c}34.32 \pm \\
0.80^{\mathrm{a}}\end{array}$ & $\begin{array}{c}33.60 \pm \\
0.58^{\mathrm{a}, \mathrm{b}}\end{array}$ \\
\hline $\begin{array}{c}\text { WBCs } \\
\left(\text { th }^{\prime} \mathbf{m m}^{3}\right)\end{array}$ & $\begin{array}{c}7.14 \pm \\
0.02^{\mathrm{d}}\end{array}$ & $\begin{array}{c}8.32 \pm \\
0.22^{\mathrm{b}}\end{array}$ & $\begin{array}{c}8.68 \pm \\
0.05^{\mathrm{a}}\end{array}$ & $\begin{array}{l}7.44 \pm \\
0.11^{\mathrm{c}}\end{array}$ & $\begin{array}{l}7.50 \pm \\
0.03^{c}\end{array}$ & $\begin{array}{l}7.48 \pm \\
0.02^{\mathrm{c}}\end{array}$ & $\begin{array}{l}7.44 \pm \\
0.06^{\mathrm{c}}\end{array}$ & $\begin{array}{c}7.43 \pm \\
0.04^{\mathrm{c}}\end{array}$ \\
\hline $\begin{array}{l}\text { Platelets } \\
\left(\text { th/mm } \mathbf{m m}^{3}\right)\end{array}$ & $\begin{array}{l}422.60 \pm \\
6.04^{\mathrm{a}, \mathrm{b}, \mathrm{c}}\end{array}$ & $\begin{array}{c}279.40 \pm \\
5.09^{\mathrm{d}}\end{array}$ & $\begin{array}{c}435.80 \pm \\
7.08^{\mathrm{a}}\end{array}$ & $\begin{array}{c}416.40 \pm \\
4.67^{\mathrm{b}, \mathrm{c}}\end{array}$ & $\begin{array}{c}412.40 \pm \\
3.74^{\mathrm{c}}\end{array}$ & $\begin{array}{c}415.20 \pm \\
4.87^{\mathrm{b}, \mathrm{c}}\end{array}$ & $\begin{array}{c}416.60 \pm \\
5.24^{\mathrm{b}, \mathrm{c}}\end{array}$ & $\begin{array}{c}431.00 \pm \\
1.70^{\mathrm{a}, \mathrm{b}}\end{array}$ \\
\hline
\end{tabular}

Values are expressed as means \pm standard errors for seven rats.

abcd $=$ values in same raw with different small letters are significantly different at $(\mathrm{P}<0.05)$. 


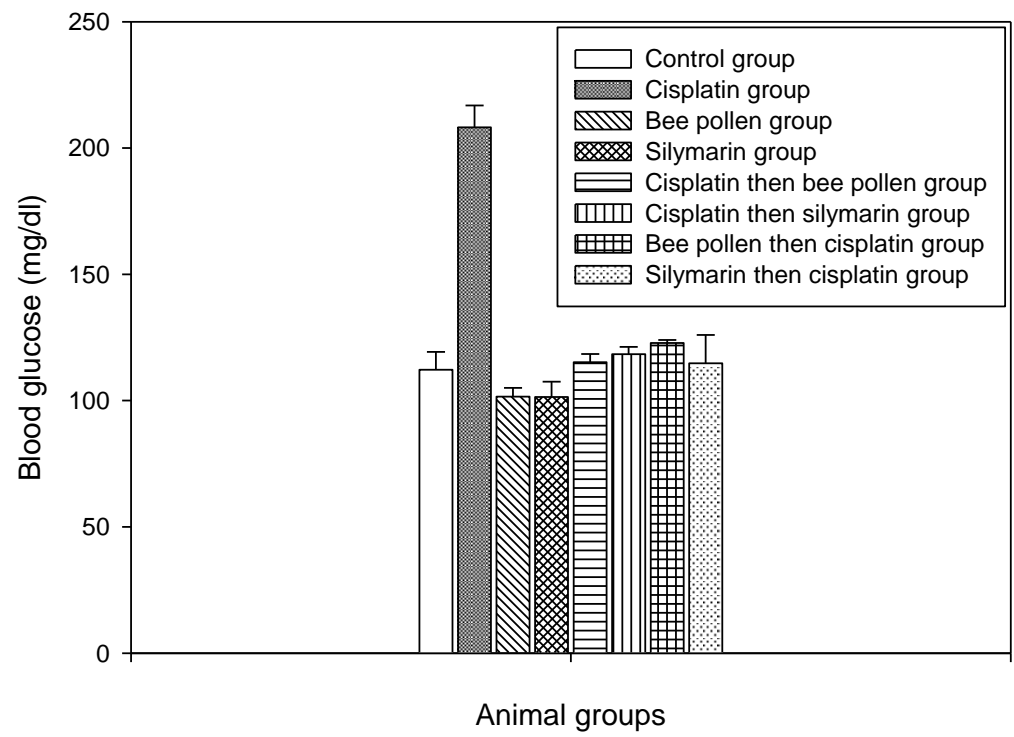

Figure (1): Effects of bee pollen and silymarin on blood glucose in different experimental groups.

\section{DISCUSSION}

Many studies have been shown that CP cause nephrotoxicity (Pazhayattil and Shirali, 2014) that is associated with severe renal dysfunction (Lin et al., 2018). Our results are consistent with studies where the kidney function parameters (urea, uric acid, and creatinine) in the $\mathrm{CP}$ group were significantly high which confirmed the occurrence of renal dysfunction. These increases in the kidney function parameters might be due to the decrease in the glomerular filtration rate or due to the oxidative stress induced by CP (Sen et al., 2018).

It was noted that bee pollen and silymarin were the causes in the marked reductions in levels of kidney function parameters in the $\mathrm{CP}$ group. The protective effect of bee pollen against CP toxicity was reported by Kocot et al. (2018). The antioxidant and detoxification effects of bee pollen were related to its content of phenolic compounds and amino acid (Nascimento and Luz Jr, 2018). Ghaznavi et al. (2016) were returned the renoprotective effect of silymarin to its ability to increase antioxidant enzymes and reducing free radical damage effects. Not only that but it also contains flavonoid complex that enables it to react as a powerful antioxidant against nephrotoxicity drugs (Sedighifard et al., 2016).

The significant decrease in serum proteins (total protein, albumin, and globulin) confirmed the renal dysfunction state in the CP group. Miyoshi et al. (2016) explained that hypoalbuminemia which is correlated with cisplatin-nephrotoxicity might be due to the binding of $\mathrm{CP}$ to plasma proteins, especially albumin. Moreover, low total proteins and albumin levels in the $\mathrm{CP}$ group could be due also to hepatotoxicity induced by CP (Nasr, 2014).

Bee pollen and silymarin were the causes in improving the protein levels in CP group. Salem et al. (2017) attributed this improvement to the ability of silymarin to regenerates hepatocytes destroyed by CP. Huang et al. (2017) showed that bee pollen has a hepatoprotective effect on liver injury induced by CP.

The CP group showed a high level of blood glucose. This result was confirmed by Jacob and Chowdhury (2015) who reported that CP causes hyperglycemia. This hyperglycemia might be related to reducing glucose transport activity and decreasing expression of the glucose transporter (GLUT4) that induced by CP (Aldalou, 2016). Meng et al. (2016) reported that silymarin decreased hyperglycemia in type 2 diabetes patient by improving $\beta$-cell function.

In the normal condition, the proximal tubule reabsorbs the most amount of filtered $\mathrm{HCO}_{3}{ }^{-}$(Skelton et al., 2010). But some drugs such as CP caused proximal RTA (Kitterer et al., 2015), which characterized by the decreasing of $\mathrm{HCO}_{3}{ }^{-}$ reabsorption rate in the proximal tubule (Soleimani and Rastegar, 2016). This may confirme our finding that the decline in the levels of arterial and venous $\mathrm{pH}, \mathrm{HCO}_{3}{ }^{-}, \mathrm{TCO}_{2}$, and $\mathrm{BE}$ in the $\mathrm{CP}$ group indicated the occurrence of proximal RTA. While the decline in $\mathrm{PCO}_{2}$ might be due to the hyperventilation which followed the acidosis state as a respiratory compensation. It was noted that hyperventilation increases $\mathrm{pH}$ in patients with metabolic acidosis (Seifter and Chang, 2016). Bee pollen and silymarin improve the proximal RTA and that appeared through increasing $\mathrm{pH}$ values. 
The reduction in $\mathrm{Hb}$ content, Hct value, RBCs count in $\mathrm{CP}$ group is a marker for anemia which can be explained through different mechanisms. one of these mechanisms is the reduction of erythropoietin (stimulates the production of red blood cells by the bone marrow) production due to the kidney damage caused by CP (Rajasekar and Jeyaprakash, 2016). The link of RBC membrane by the free radical produced by $\mathrm{CP}$ which resulting in lowering of the $\mathrm{Hb}$ level might be another mechanism for anemia induced by CP (Janakiraman and Jeyaprakash, 2015). While Aldalou (2016) reported that the destruction of bone marrow cells, suppression of the activity of hematopoietic tissues, impaired erythropoiesis, and accelerated $\mathrm{RBCs}$ destruction by $\mathrm{CP}$ might be reasons for anemia induced by $\mathrm{CP}$.

It turned out that bee pollen reduced anemia caused by CP. The anti-anemia effect of bee pollen might be returned to improving the absorption and utilization of nutritional iron (Rzepecka-Stojko et al., 2015). A higher content of vitamin $\mathrm{C}$ in bee pollen also increases $\mathrm{Hb}$ content and RBCs count (KomosinskaVassev et al., 2015). While silymarin prevents hemolysis by reducing the rate and the total content of glutathione loss in erythrocytes (Karimi et al., 2011).

\section{CONCLUSION}

Results concluded that the bee pollen and silymarin are able to ameliorate the acid-base balance in the kidney which was disturbed by nephrotoxicity induced by CP. Not only that but they also have powerful effects against anemia that caused by $\mathrm{CP}$.

\section{REFERENCES}

Abouzeinab, N.S. (2015): Antioxidant effect of silymarin on cisplatin-induced renal oxidative stress in rats. J. Pharmacol. Toxicol., 10: 1-19.

Aldalou, A.R. (2016): Amelioration of cisplatininduced toxicity in blood domestic rabbit's by sodium thiosulfate or ginger extract. European j. biomed. pharm. sci., 3 (7): 578-586.

Ghaznavi, H.; Mehrzadi, S.; Dormanesh, B.; Tabatabaei, S.M.; Vahedi, H.; Hosseinzadeh, A.; Pazoki-Toroudi, H. and Rashidian, A. (2016): Comparison of the protective effects of melatonin and silymarin against gentamicin-induced nephrotoxicity in rats. J. Evid. Based Complementary Altern. Med., 21: NP49-55.

Hall, A.M.; Bass, P. and Unwin, R.J. (2014): Druginduced renal Fanconi syndrome. Q. J. Med., 107(4): 261-269.

Hayati, F.; Hossainzadeh, M.; Shayanpour, S.; Abedi-Gheshlaghi, Z. and Beladi Mousavi, S.S. (2016): Prevention of cisplatin nephrotoxicity. J. Nephropharmacol., 5(1): 57-60.

Hosseini, A.; Fanoudi, S.; Mollazadeh, H.; Aghaei, A. and Boroushaki, M.T. (2018): Protective effect of Rheum turkestanicum against cisplatin by reducing oxidative stress in kidney tissue. $\mathrm{J}$. Pharm. Bioallied. Sci, 10(2): 66-71.

Huang, H.; Shen, Z.; Geng, Q.; Wu, Z.; Shi, P. and Miao, X. (2017): Protective effect of Schisandra chinensis bee pollen extract on liver and kidney injury induced by cisplatin in rats. Biomed. Pharmacother., 95: 1765-1776.

Jacob, P. and Chowdhury, T.A. (2015): Management of diabetes in patients with cancer. Q. J. Med., 108: 443-448.

Janakiraman, $M$. and Jeyaprakash, K. (2015): Nephroprotective effect of Vitex negundo linn on cisplatin induced nephrotoxicity in male albino rats. World J. Pharm. Sci., 3(4): 706715.

Karimi, G.; Vahabzadeh, M.; Lari, P.; Rashedinia, M. and Moshiri, M. (2011): "Silymarin", a promising pharmacological agent for treatment of diseases. Iran J Basic Med Sci., 14(4): 308317.

Kitterer, D.; Schwab, M.; Alscher, M.D.; Braun, N. and Latus, J. (2015): Drug-induced acid-base disorders. Pediatr. Nephrol., 30: 1407-1423.

Kocot, J.; Kiełczykowska, M.; Luchowska-Kocot, D.; Kurzepa, J. and Musik, I. (2018): Antioxidant Potential of Propolis, Bee Pollen and Royal Jelly: Possible Medical Application. Oxid. Med. Cell Longev., 2018: 7074209.

Komosinska-Vassev, K.; Olczyk, P.; Kaźmierczak, J.; Mencner, L. and Olczyk, K. (2015): Bee pollen: chemical composition and therapeutic application. Evid. Based Complement. Alternat. Med., 2015: ID 297425.

Lin, M.T.; Ko, J.L.; Liu, T.C.; Chao, P.T. and Ou, C.C. (2018): Protective effect of D-methionine on body weight loss, anorexia, and nephrotoxicity in cisplatin-induced chronic toxicity in rats. Integr. Cancer Ther., 17(3): 813-824.

Mansour, H.H.; Hafez H.F. and Fahmy, N.M. (2006): Silymarin modulates cisplatin-induced oxidative stress and hepatotoxicity in rats. J. Biochem. Mol. Biol., 39(6): 656-661.

Meng, R.; Mahadevan, J.; Oseid, E.; Vallerie, S. and Robertson, R.P. (2016): Silymarin activates cAMP phosphodiesterase and stimulates insulin secretion in a glucose-dependent manner in HIT-T15 cells. Antioxidants. 5: 47.

Miyoshi, T.; Misumi, N.; Hiraike, M.; Mihara, Y.; Nishino, T.; Tsuruta, M.; Kawamata, Y.; Hiraki, Y.; Kozono, A. and Ichiki, M. (2016): Risk factors associated with cisplatin-induced nephrotoxicity in patients with advanced lung cancer. Biol. Pharm. Bull., 39 (12): 20092014. 
Mohamed, W.R.; Arafa, E.A.; Shehata, B.A.; El Sherbiny, G.A. and Elgendy, A.A.M. (2015): Beneficial effects of hesperidin against cisplatin-induced nephrotoxicity and oxidative stress in rats. Br. J. Pharmacol. Toxicol., 6(3): 56-63.

Nascimento, A.M.C.B. and Luz Jr, G.E. (2018): Bee pollen properties: uses and potential pharmacological applications-a review. J. Anal Pharm. Res., 7(4): 513-515.

Nasr, A.Y. (2014): Protective effect of aged garlic extract against the oxidative stress induced by cisplatin on blood cells parameters and hepatic antioxidant enzymes in rats. Toxicol. Rep., 1: 682-691.

Pazhayattil, G.S. and Shirali, A.C. (2014): Druginduced impairment of renal function. Int. J. Nephrol. Renovasc. Dis., 7: 457-468.

Philip, P. and Jacob, C.S. (2018): Proximal renal tubular acidosis with primary Fanconi syndrome. Int. J. Contemp. Pediatr., 5(3): 1131-1133.

Rajasekar, R. and Jayaprakash, K. (2016): Protective effect of Agaricus bisporus (Button mushroom) on cisplatin induced nephrotoxicity in albino rats. World J. Pharm. Sci., 4(9): 366-372.

Rzepecka-Stojko, A.; Stojko, J.; Kurek-Górecka, A.; Górecki, M.; Kabała-Dzik, A.; Kubina, R.; Moździerz, A. and Buszman, E. (2015): Polyphenols from bee pollen: structure, absorption, metabolism and biological activity. Molecules. 20(12): 21732-21749.
Salem, S.I.; Abd El-Baky, A.A. and Mohammed, F.F. (2017): Cytoprotective effect of silymarin on cisplatin-induced hepatotoxicity and bone marrow toxicity in rats. Asian J. Anim. Sci., 11(3): 140-152.

Sedighifard, Z.; Roghani, F.; Bidram, P.; Harandi, S.A. and Molavi, S. (2016): Silymarin for the prevention of contrast-induced nephropathy: a placebo-controlled clinical trial. Int. J. Prev. Med., 22: 7- 23.

Seifter, J.L. and Chang. H.Y. (2016): Disorders of acid-base balance: new perspectives. Kidney Dis., 2: 170-186.

Sen, S.; Chakraborty, R. and Kalita, P. (2018): Dillenia indica fruit prevents cisplatin-induced kidney injury in experimental rats through modulation of oxidative stress, marker enzyme, and biochemical changes. Nutrire. 43: 15.

Skelton, L.A.; Boron, W.F. and Zhou, Y. (2010): Acid-base transport by the renal proximal tubule. J. Nephrol., 23: S4-18.

Soleimani, M. and Rastegar, A. (2016): Pathophysiology of renal tubular acidosis: core curriculum. Am. J. Kidney Dis., 68: 488-498.

Yaxley, J. and Pirrone, C. (2016): Review of the diagnostic evaluation of renal tubular acidosis. Ochsner. J., 16: 525-530.

Yildiz, O.; Can, Z.; Sarai, O.; Yulug, E.; Öztörk, F.; Aliyazicioğlu, R.; Canpolat, S. and Kolayli, S. (2013): Hepatoprotective potential of chesnut bee pollen on carbon tetrachloride-induced hepatic damages in rats. Evid. Based Complement Alternat. Med., 2013: 461-478.

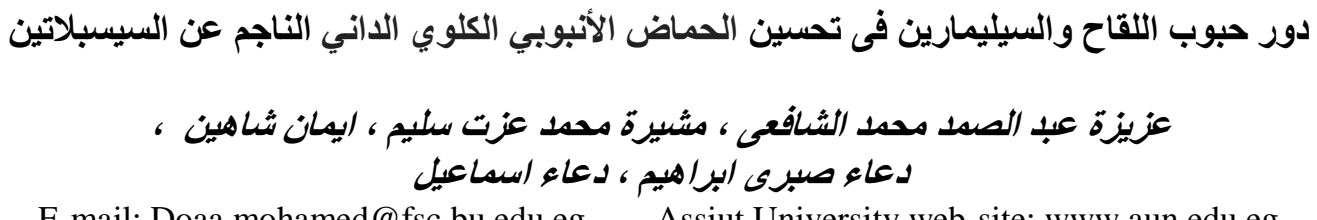

E-mail: Doaa.mohamed@fsc.bu.edu.eg Assiut University web-site: www.aun.edu.eg

الحماض الأنبوبي الكلوي الداني (RTA) هو خلل في توازن الحمض القاعدي في الكلى بفقد أيونات البيكربونات في البول. وأحد

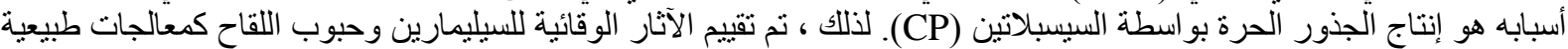

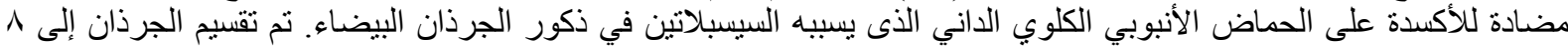

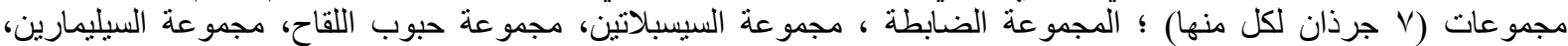

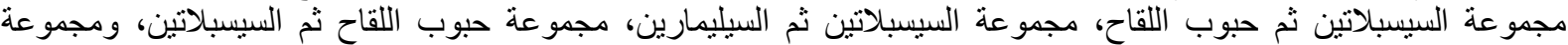

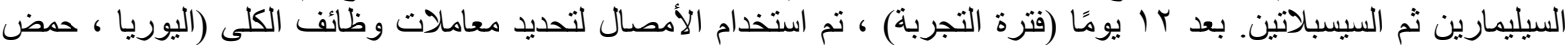

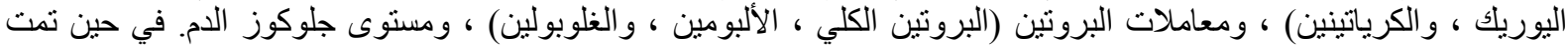

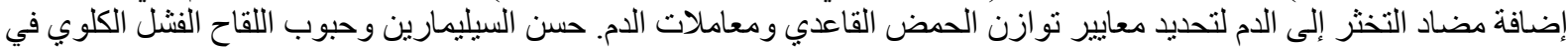

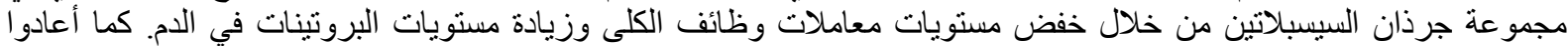

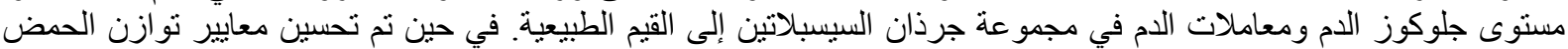

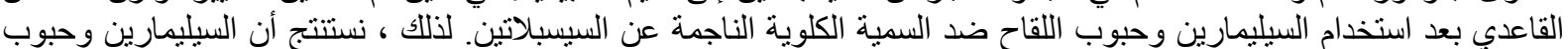

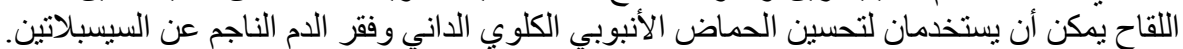

